\title{
Pacific
}

Journal of

Mathematics

\section{CONVERGENCE OF INFINITE EXPONENTIALS}

\author{
GENNADY BACHMAN
}




\title{
CONVERGENCE OF INFINITE EXPONENTIALS
}

\author{
GenNADY BACHMAN
}

In this paper we give two tests of convergence for an infinite exponential $a_{1}^{a_{2}^{a_{3}}}$. We also show that these tests are essentially the best possible.

1. Introduction and Statement of Results. Given a sequence of positive real numbers $a_{n}, n=1,2,3, \ldots$, we associate with it a sequence of partial exponentials $E_{n}, n=1,2,3, \ldots$, defined by

$$
E_{n}=a_{1}^{a_{2}^{a^{a_{n}}}}
$$

We will call $\left\{a_{n}\right\}$ a sequence of exponents and the sequence $\left\{E_{n}\right\}$ an infinite exponential. As in the study of sums and products one would like to develop tests of convergence of an infinite exponential. Euler $[\mathbf{E}]$ was the first to give such a test. He showed that in the special case $a_{1}=a_{2}=a_{3}=\cdots=a, E_{n}$ is convergent if and only if $e^{-e} \leq a \leq e^{1 / e}$. This result has been rediscovered by many authors. An extensive bibliography of papers containing this and related results may be found in the survey paper by Knoebel [K].

In the general case of non-constant exponents the best known results are due to Barrow $[\mathbf{B}]$. He showed (although some of his arguments are rather sketchy) that $\left\{E_{n}\right\}$ is convergent for $e^{-e} \leq$ $a_{n} \leq e^{1 / e}, n \geq n_{0}$. He also considered the cases $a_{n} \geq e^{1 / e}$ and $a_{n} \leq e^{-e}$. In the first case, writing $a_{n}=e^{1 / e}+\epsilon_{n}$, with $\epsilon_{n} \geq 0$, he showed that $\left\{E_{n}\right\}$ is convergent if

$$
\lim _{n \rightarrow \infty} \epsilon_{n} n^{2}<\frac{e^{1 / e}}{2 e}
$$

and is divergent if

$$
\lim _{n \rightarrow \infty} \epsilon_{n} n^{2}>\frac{e^{1 / e}}{2 e}
$$


In the second case, writing $a_{n}=e^{-e}-\epsilon_{n}$, with $\epsilon_{n} \geq 0$, he obtained the conditions $\lim _{n \rightarrow \infty} \epsilon_{n}=0$ and $\lim _{n \rightarrow \infty} n^{q} \epsilon_{n}=0$ for some $q>1$, as necessary and sufficient for the convergence of $\left\{E_{n}\right\}$ respectively.

Ramanujan made the following entry (without a proof) on page 30 of his third notebook (see [R], page 390, also posed as an unsolved problem at the 1991 West Coast Number Theory Conference, see Problem 91:06 in [G]): $E_{n}$ is convergent when

$$
1+\log \log a_{n} \leq \frac{1}{2}\left\{\frac{1}{n^{2}}+\frac{1}{(n \log n)^{2}}+\frac{1}{(n \log n \log \log n)^{2}}+\cdots\right\},
$$

and is divergent when the left hand side is greater than the right hand side with any 1 replaced by $1+\epsilon$. This statement requires some clarification. What Ramanujan probably had in mind was a test of convergence of an infinite exponential of a sequence of exponents $a_{n} \geq 1$. A sufficient condition for the convergence was furnished by the inequality $1+\log \log a_{n} \leq f(n), n \geq n_{0}$ for an appropriate, possibly any, function $f(n)$ with an asymptotic expansion given by the right hand side of (1.4) as $n \rightarrow \infty$. An easy calculation shows that Barrow's satements (1.2), even in the much stronger form with $<$ replaced by $\leq$, and (1.3) are contained in Ramanujan's assertion with the right hand side of (1.4) truncated after the first term.

The main purpose of this paper is to give a proof of Ramanujan's test of convergence of an infinite exponential and to generalize it to the case of complex exponents $a_{n}$. In order that the exponentiation be unambiguous we assume that the sequence of complex numbers $b_{n}, n=1,2,3, \ldots$ is given and set

$$
a_{n}=e^{b_{n}} .
$$

With this definition of the sequence $\left\{a_{n}\right\}(1.1)$ is well defined. The case of complex exponents has also been considered before. The best known results here are due to Shell $[\mathbf{S}]$, in the case of equal exponenents, and Thron [T], in the general case. We state here. only the results of Thron, who showed that $\left\{E_{n}\right\}$ is convergent if $\left|b_{n}\right| \leq 1 / e, n \geq n_{0}$. We first give the following test of convergence of an infinite exponential with complex exponents:

THEOREM 1. Let $\left\{a_{n}\right\}$ and $\left\{E_{n}\right\}$ be defined by (1.5) and (1.1) 
respectively. Now set

$$
\hat{a}_{n}=e^{\left|b_{n}\right|} \quad[n \geq 1]
$$

and define $\hat{E}_{n}, n=1,2,3, \ldots$, by (1.1) in terms of the sequence $\left\{\hat{a}_{n}\right\}$. Then if $\hat{E}_{n}$ converges, then so must $E_{n}$.

The above test of convergence is of independent interest. In particular, Thron's result follows immediately from Barrow's results for real exponents $a_{n}, 1 \leq a_{n} \leq e^{1 / e}$, and Theorem 1 .

To state our results concerning Ramanujan's test of convergence we introduce the following notation for the iterated logarithm. Setting $x_{1}=e$ and

$$
L_{1}(x)=L(x)=\log (x), \quad \text { for } x \geq e,
$$

we define recursively $x_{k}$ and $L_{k}(x)$, for $k \geq 2$, by $x_{k}=e^{x_{k-1}}$, and

$$
L_{k}(x)=L_{k-1}(L(x)), \quad \text { for } x \geq x_{k} .
$$

With this notation we have:

THEOREM 2. Let $\left\{E_{n}\right\}$ be defined by (1.5) and (1.1) respectively. Then the infinite exponential converges if there exist positive integers $k_{0}$ and $n_{0}$ such that for all $n \geq n_{0}$ we have

$$
\begin{aligned}
& 1+\log \left|\log a_{n}\right|=1+\log \left|b_{n}\right| \\
& \leq \frac{1}{2}\left\{\frac{1}{n^{2}}+\frac{1}{\left(n L_{1}(n)\right)^{2}}+\frac{1}{\left(n L_{1}(n) L_{2}(n)\right)^{2}}+\cdots\right. \\
& \left.\quad+\frac{1}{\left(n L_{1}(n) L_{2}(n) \cdots L_{k_{0}}(n)\right)^{2}}\right\}
\end{aligned}
$$

To complement this result we prove:

THEOREM 3. Let $E_{n}$ be defined by (1.1) in terms of a sequence of real numbers $a_{n}$ satisfying $a_{n}>1$ and

$$
\begin{aligned}
& 1+\log \log a_{n} \\
& \geq \frac{1}{2}\left\{\frac{1}{n^{2}}+\frac{1}{\left(n L_{1}(n)\right)^{2}}+\cdots\right. \\
& \left.\quad+\frac{1}{\left(n L_{1}(n) L_{2}(n) \cdots L_{k_{0}-1}(n)\right)^{2}}+\frac{1+\epsilon}{\left(n L_{1}(n) L_{2}(n) \cdots L_{k_{0}}(n)\right)^{2}}\right\}
\end{aligned}
$$

for $n \geq n_{0}$, for some positive integers $k_{0}$ and $n_{0}$, and $\epsilon>0$. Then the infinite exponential diverges. 
2. Preliminaries. In this section we prove three lemmas. The first of these reduces the principal case of our problem to an equivalent problem which is easier to handle. We will find it convenient to use the notation

$$
\left[x_{1}, x_{2}, \ldots, x_{n}\right]=x_{1}^{x_{2}^{x_{n}}} \quad \text { and } \quad\left[x_{1}, x_{2}, x_{3}, \ldots\right]
$$

to denote partial exponents and an infinite exponential respectively. We also set

(2.1) $l_{0}(x)=\frac{1}{x}$ and $l_{k}(x)=\frac{1}{x L_{1}(x) L_{2}(x) \cdots L_{k}(x)} \quad[k \geq 1]$.

LEMMA 1. Let a sequence of real numbers $x_{n}, n=1,2,3, \ldots$, satisfying $x_{n}>1$ be given. Define a sequence $X_{n}, n=1,2,3, \ldots$ by

$$
x_{n}=\exp \left(\frac{1+X_{n}}{e}\right) \text {. }
$$

Then $\left[x_{1}, x_{2}, x_{3}, \ldots\right]$ converges if and only if there exists a sequence $Y_{n}, n=1,2,3, \ldots$, satisfying $Y_{n} \geq-1$ and such that the inequality

$$
1+Y_{n} \geq\left(1+X_{n}\right) e^{Y_{n+1}}
$$

holds.

Proof. Since $x_{n}>1$ the sequence $\left[x_{1}, x_{2}, x_{3}, \ldots\right]$ is monotonically increasing. Hence to show that it is convergent it suffices to show that it is bounded. But this follows immediately from (2.2) and (2.3) since

$$
\left[x_{1}, x_{2}, \ldots, x_{n}\right] \leq\left[x_{1}, x_{2}, \ldots, x_{n}, e^{1+Y_{n+1}}\right] \leq e^{1+Y_{1}} .
$$

In the opposite direction suppose that the infinite exponential $\left[x_{1}, x_{2}, x_{3}, \ldots\right]$ is convergent. Since $x_{n}>1$ then so must be an infinite exponential $\left[x_{n}, x_{n+1}, x_{n+2}, \ldots\right]$ for any $n \geq 1$. Denoting a limit of $\left[x_{n}, x_{n+1}, x_{n+2}, \ldots\right]$ by $e^{1+Y_{n}}$, we observe that $Y_{n} \geq-1$ and that the sequence $\left\{Y_{n}\right\}$ satisfies

$$
e^{1+Y_{n}}=\left[x_{n}, e^{1+Y_{n+1}}\right]=e^{\left(1+X_{n}\right) e^{Y_{n+1}}} .
$$

This gives (2.3) with equality and completes the proof of the lemma. 
The next two lemmas are the main ingredients in the proofs of Theorems 2 and 3.

LEMMA 2. Let $T_{n}^{k}, C_{n}^{k}$ and $X_{n}^{k}$ be defined by

$$
\begin{aligned}
& T_{n}^{k}=\sum_{j=0}^{k} l_{j}(n-1), \\
& C_{n}^{k}=\frac{1}{2} \sum_{j=0}^{k} l_{j}^{2}(n),
\end{aligned}
$$

and

$$
1+X_{n}^{k}=\left(1+T_{n}^{k}\right) e^{-T_{n+1}^{k}}
$$

where $l_{j}(n)$ is given by (2.1), for any integers $k \geq 0$ and $n \geq 2$ for which the right hand sides of (2.4) and (2.5) are defined. Then there exists a sequence of integers $\left\{n_{k}\right\}$ such that for all $n \geq n_{k}$ we have

$$
C_{n}^{k}<X_{n}^{k}<C_{n}^{k+1}
$$

Proof. Let an integer $k \geq 0$ be fixed. We write $T_{n}$ and $X_{n}$ to denote $T_{n}^{k}$ and $X_{n}^{k}$ respectively. By (2.4) and (2.1) we have $T_{n}=$ $O_{k}(1 / n)<1$, for $n \geq n_{k}$ sufficiently large in terms of $k$. For such integers $n$ we can expand the right hand side of (2.6) in a Taylor series to obtain

$$
\begin{aligned}
1+X_{n}= & \left(1+T_{n}\right) e^{-T_{n+1}} \\
= & \left(1+T_{n}\right)\left(1-T_{n+1}+\frac{1}{2}\left(T_{n+1}\right)^{2}-\frac{1}{6}\left(T_{n+1}\right)^{3}+O_{k}\left(\frac{1}{n^{4}}\right)\right) \\
= & 1+T_{n}-T_{n+1}+\frac{1}{2}\left(T_{n+1}\right)^{2}-T_{n} T_{n+1}+\frac{1}{2} T_{n}\left(T_{n+1}\right)^{2} \\
& \quad-\frac{1}{6}\left(T_{n+1}\right)^{3}+O_{k}\left(\frac{1}{n^{4}}\right) .
\end{aligned}
$$

Now, by (2.4) and (2.1), expanding $T_{n}$ about $n+1$ we get

$$
T_{n}=T_{n+1}-T_{n+1}^{\prime}+\frac{1}{2} T_{n+1}^{\prime \prime}-\frac{1}{6} T_{\xi}^{\prime \prime \prime}
$$


for some $\xi$ with $n<\xi<n+1$, where

$$
\begin{aligned}
T_{n+1}^{\prime}=\sum_{j=0}^{k} l_{j}^{\prime}(n)=-\sum_{j=0}^{k} l_{j}(n) \sum_{i=0}^{j} l_{i}(n), \\
T_{n+1}^{\prime \prime}=\sum_{j=0}^{k} l_{j}^{\prime \prime}(n)=-\left(\left(l_{0}^{2}(n)\right)^{\prime}+\sum_{j=1}^{k} \sum_{i=0}^{j}\left(l_{j}(n) l_{i}(n)\right)^{\prime}\right) \\
=\frac{2}{n^{3}}+O_{k}\left(\frac{1}{n^{3} \log n}\right),
\end{aligned}
$$

and

$$
T_{\xi}^{\prime \prime \prime}=\sum_{j=0}^{k} l_{j}^{\prime \prime \prime}(\xi-1)=O_{k}\left(\frac{1}{n^{4}}\right) .
$$

Substituting (2.9)-(2.12) into (2.8) and simplifying the resulting expression we obtain

$$
1+X_{n}=1-T_{n+1}^{\prime}-\frac{1}{2}\left(T_{n+1}\right)^{2}+\frac{1}{3 n^{3}}+O_{k}\left(\frac{1}{n^{3} \log n}\right) .
$$

Hence by (2.10), (2.4) and (2.5) we have

$$
\begin{aligned}
X_{n} & =-T_{n+1}^{\prime}-\frac{1}{2}\left(T_{n+1}\right)^{2}+\frac{1}{3 n^{3}}+O_{k}\left(\frac{1}{n^{3} \log n}\right) \\
& =\frac{1}{2} \sum_{j=0}^{k} l_{j}^{2}(n)+\frac{1}{3 n^{3}}+O_{k}\left(\frac{1}{n^{3} \log n}\right) \\
& =C_{n}^{k}+\frac{1}{3 n^{3}}+O_{k}\left(\frac{1}{n^{3} \log n}\right) .
\end{aligned}
$$

This, for $n \geq n_{k}$ sufficiently large in terms of $k$, implies (2.7) and completes the proof of the lemma.

LEMMA 3. Let $T_{n}^{k}$ and $X_{n}^{k}$ be defined by (2.4) and (2.6) of Lemma 2. Moreover, let $x_{n}^{k}$ be defined by

$$
x_{n}^{k}=\exp \left\{\frac{1+X_{n}^{k}}{e}\right\} \text {. }
$$


Then we have

$$
\lim _{m \rightarrow \infty}\left[x_{n}^{k}, x_{n+1}^{k}, \ldots, x_{m}^{k}\right]=e^{1+T_{n}^{k}}
$$

Proof. We begin by observing that it suffices to show that there exists a sequence of integers $\left\{n_{k}^{\prime}\right\}$ such that (2.15) holds for all $n \geq n_{k}^{\prime}$. Indeed, assuming this we have, for any $l \geq n_{k}^{\prime}$,

$$
\begin{aligned}
\lim _{m \rightarrow \infty}\left[x_{n}^{k}, x_{n+1}^{k}, \ldots, x_{m}^{k}\right] & =\left[x_{n}^{k}, \ldots, x_{l}^{k}, \lim _{m \rightarrow \infty}\left[x_{l+1}^{k}, \ldots, x_{m}^{k}\right]\right] \\
& =\left[x_{n}^{k}, \ldots, x_{l}^{k}, e^{1+T_{l+1}^{k}}\right] \\
& =e^{1+T_{n}^{k}}
\end{aligned}
$$

by (2.14) and (2.6). To exhibit the existence of such a sequence $\left\{n_{k}^{\prime}\right\}$ we first observe that by (2.14), Lemma 1 and Lemma 2, any infinite exponential $\left[x_{n}^{k}, x_{n+1}^{k}, x_{n+2}^{k}, \ldots\right]$, with $n \geq n_{k}$, where $\left\{n_{k}\right\}$ is a sequence defined in the statement of Lemma 2 , is convergent. Let us denote the limit of such an infinite exponential by $e^{1+S_{n}^{k}}$. Then (2.15) will follow if we can show that

$$
S_{n}^{k}=T_{n}^{k}
$$

for all $n \geq n_{k}^{\prime} \geq n_{k}$ sufficiently large in terms of $k$.

To this end let us define, for integers $k \geq 0$ and $n \geq n_{k}, t_{n}^{k}$ by

$$
t_{n}^{k}=T_{n}^{k}-S_{n}^{k}
$$

We will deduce (2.16) from the following three inequalities:

$$
S_{n}^{k}>S_{n}^{l}>0 \quad\left[k>l ; n \geq \max \left(n_{k}, n_{l}\right)\right],
$$

$$
t_{n}^{k} \geq 0
$$

and

(2.20) $t_{m}^{k} \geq t_{n}^{k}\left(\frac{L_{k}(m-1)}{L_{k}(n)}\right)^{t_{n}^{k} / 2} l_{k}(m-1) \quad\left[m>n \geq n_{k}^{\prime}\right]$,

with $n_{k}^{\prime} \geq n_{k}$ sufficiently large in terms of $k$, where in the case $k=0 L_{0}(x)=x$. Indeed, assume (2.16) fails with $k=0$ and some 
$n \geq n_{0}^{\prime}$. Then by (2.19) $t_{n}^{0}>0$, and hence by (2.20) and (2.4) we get

$$
t_{m}^{0} \geq t_{n}^{0}\left(\frac{m-1}{n}\right)^{t_{n}^{0} / 2} l_{0}(m-1)>l_{0}(m-1)=T_{m}^{0},
$$

for some $m>n$ sufficiently large in terms of $t_{n}^{0}$. But by (2.17) this implies that $S_{m}^{0}<0$, which contradicts (2.18). Thus (2.16), with $k=0$, must hold for all $n \geq n_{0}^{\prime}$. We now proceed by induction on $k$. Assume that (2.16) fails for some $k>0$ and $n \geq n_{k}^{\prime}$. Arguing as above we obtain the inequality

$$
t_{m}^{k}>l_{k}(m-1),
$$

for some $m>n$ sufficiently large in terms of $t_{n}^{k}$. This, together with (2.17) and (2.4) yield

$$
S_{m}^{k}=T_{m}^{k}-t_{m}^{k}<T_{m}^{k-1}=S_{m}^{k-1},
$$

by the inductive hypothesis, provided $m \geq n_{k-1}^{\prime}$, as we may assume. But since (2.21) contradicts (2.18) we conclude that (2.16) and hence (2.15) hold. Therefore it only remains to prove (2.18)-(2.20).

To this end, assuming as we may that the sequence $\left\{n_{k}\right\}$ is increasing, we have, for $k>l$ and $n \geq n_{k} \geq n_{l}$,

$$
X_{n}^{k}>X_{n}^{l}>0 \text {, }
$$

and hence

$$
x_{n}^{k}>x_{n}^{l}>e^{1 / e},
$$

by (2.7), (2.5) and (2.14). It was already shown by Euler $[\mathbf{E}]$ that the infinite exponential with constant exponents $e^{1 / e}$ converges to $e$. This fact together with (2.22) yields (2.18). To prove (2.19) we observe that for $m>n \geq n_{k}$, we have

$$
\left[x_{n}^{k}, x_{n+1}^{k}, \ldots, x_{m}^{k}\right]<\left[x_{n}^{k}, \ldots, x_{m}^{k}, e^{1+T_{m+1}^{k}}\right]=e^{1+T_{n}^{k}},
$$

by (2.14) and (2.6). Hence $S_{n}^{k} \leq T_{n}^{k}$ and thus (2.19) holds by the definition (2.16) of $t_{n}^{k}$.

We begin proving (2.20) by observing that by the definition of $S_{n}^{k}$ and (2.14) we have

$$
e^{1+S_{n}^{k}}=\left[x_{n}^{k}, e^{1+S_{n+1}^{k}}\right]=e^{\left(1+X_{n}^{k}\right) e^{S_{n+1}^{k}}} .
$$


Hence $S_{n}^{k}$ satisfies the identity (2.6) with $T_{n}^{k}$ replaced by $S_{n}^{k}$. Let us fix $k$ and write $S_{n}, T_{n}$ and $t_{n}$ for $S_{n}^{k}, T_{n}^{k}$ and $t_{n}^{k}$ respectively. From our last observation it follows that

$$
\left(1+S_{n}\right) e^{-S_{n+1}}=\left(1+T_{n}\right) e^{-T_{n+1}} .
$$

Substituting $S_{m}=T_{m}-t_{m}, m=n, n+1$, into the last identity leads to

$$
\frac{t_{n}}{1+T_{n}}=1-e^{-t_{n+1}}
$$

Now, by (2.19), (2.18), (2.4) and (2.1), we have

$$
0 \leq t_{n} \leq T_{n} \ll_{k} \frac{1}{n}
$$

Hence

$$
\begin{aligned}
1-e^{-t_{n+1}} & =t_{n+1} \sum_{i=1}^{\infty} \frac{1}{i !}\left(-t_{n+1}\right)^{i-1} \\
& <t_{n+1} \sum_{i=1}^{\infty}\left(-\frac{t_{n+1}}{2}\right)^{i-1}=\frac{t_{n+1}}{1+t_{n+1} / 2}
\end{aligned}
$$

provided $n \geq n_{k}^{\prime}$ sufficiently large in terms of $k$. Using this bound for the right hand side of (2.23) we obtain

$$
t_{n+1}>t_{n} \frac{1+t_{n+1} / 2}{1+T_{n}} .
$$

It now follows that for any integers $m>n \geq n_{k}^{\prime}$ we have

$$
t_{m}>t_{n} \prod_{i=n}^{m-1} \frac{1+t_{i+1} / 2}{1+T_{i}}
$$

We use (2.25) in two steps. Firstly, by (2.25) and (2.24), we have

$$
\begin{aligned}
t_{m} & >t_{n} \prod_{i=n}^{m-1} \frac{1}{1+T_{i}}=t_{n} \exp \left\{\sum_{i=n}^{m-1} \log \frac{1}{1+T_{i}}\right\} \\
> & t_{n} \exp \left\{-\sum_{i=n}^{m-1} T_{i}\right\}
\end{aligned}
$$


for $m>n>\geq n_{k}^{\prime}$ sufficiently large in terms of $k$. Now, by (2.4) and $(2.1)$,

$$
\sum_{i=n}^{m-1} T_{i}=\sum_{i=n}^{m-1} \sum_{j=0}^{k} l_{j}(i-1)<\sum_{j=0}^{k} \int_{n-2}^{m-1} l_{j}(x) d x<\sum_{j=0}^{k} L_{j+1}(m-1) .
$$

Hence

$$
\begin{aligned}
t_{m} & >t_{n} \exp \left\{-\sum_{j=0}^{k} L_{j+1}(m-1\}\right. \\
& =t_{n} \frac{1}{(m-1) L_{1}(m-1) \ldots L_{k}(m-1)} \\
& =t_{n} l_{k}(m-1)
\end{aligned}
$$

for any integers $m>n \geq n_{k}^{\prime}$. We now reiterate the abpve argument this time using (2.26) instead of (2.19) on the right hand side of (2.25). To this end we observe that for $m>n \geq n_{k}^{\prime}$ sufficiently large in terms of $k$ we have $t_{n} l_{k}(i) / 2<T_{i} / 2 \ll_{k} 1 / i$ and

$$
\sum_{j=n}^{m-1} l_{k}(i)>\int_{n}^{m-1} l_{k}(x) d x=L_{k+1}(m-1)-L_{k+1}(n) .
$$

Thus

$$
\begin{aligned}
t_{m} & >t_{n} \prod_{i=n}^{m-1} \frac{1+t_{n} l_{k}(i) / 2}{1+T_{i}}=t_{n} \exp \left\{\sum_{i=n}^{m-1} \log \left(\frac{1+t_{n} l_{k}(i) / 2}{1+T_{i}}\right)\right\} \\
& >t_{n} \exp \left\{\sum_{i=n}^{m-1}\left(\frac{1}{2} t_{n} l_{k}(i)-T_{i}\right)\right\} \\
& >t_{n} \exp \left\{\frac{1}{2} t_{n}\left(L_{k+1}(m-1)-L_{k+1}(n)\right)-\sum_{j=0}^{k} L_{j+1}(m-1)\right\} \\
& =t_{n}\left(\frac{L_{k}(m-1)}{L_{k}(n)}\right)^{t_{n} / 2} l_{k}(m-1) .
\end{aligned}
$$

This gives (2.20) and completes the proof of the lemma. 
3. Proofs of Theorems. Proof of Theorem 1. We may assume that for all $n, a_{n} \neq 1$, for otherwise both $\left[a_{1}, a_{2}, a_{3}, \ldots\right]$ and $\left[\hat{a}_{1}, \hat{a}_{2}, \hat{a}_{3}, \ldots\right]$ converge trivially. Now fix an integer $n$, and for $z \in C$, set

$$
f(z)=\frac{d}{d z}\left[a_{1}, a_{2}, \ldots, a_{n}, z\right]
$$

and

$$
g(z)=\frac{d}{d z}\left[\hat{a}_{1}, \hat{a}_{2}, \ldots, \hat{a}_{n}, z\right] .
$$

We have, for any $m>n$,

$$
\begin{aligned}
{\left[a_{1}, a_{2}, \ldots, a_{m}\right]-\left[a_{1}, a_{2}, \ldots, a_{n}\right] } & \\
& =\left[a_{1}, \ldots, a_{n},\left[a_{n+1}, \ldots a_{m}\right]\right]-\left[a_{1}, \ldots, a_{n}, 1\right] \\
& =\int_{1}^{\left[a_{n+1}, \ldots, a_{m}\right]} f(z) d z .
\end{aligned}
$$

Setting

$$
u=\left[a_{n+1}, \ldots, a_{m}\right],
$$

and

$$
w=\left[\hat{a}_{n+1}, \ldots, \hat{a}_{m}\right],
$$

we estimate the right hand side of (3.3) to obtain

$$
\begin{aligned}
& \left|\left[a_{1}, a_{2}, \ldots, a_{m}\right]-\left[a_{1}, a_{2}, \ldots, a_{n}\right]\right| \\
& \quad=\left|\int_{1}^{u} f(z) d z\right| \\
& \quad=\left|\int_{0}^{1} f(1+(u-1) t) d(1+(u-1) t)\right| \\
& \quad \leq|u-1| \int_{0}^{1}|f(1+(u-1) t)| d t .
\end{aligned}
$$

Now, by (3.1), (1.5), (3.2) and (1.6), we have

$$
\begin{aligned}
f(z) & =b_{1}\left[a_{1}, a_{2}, \ldots, a_{n}, z\right] \frac{d}{d z}\left[a_{2}, a_{3}, \ldots, a_{n}, z\right] \\
& =\prod_{k=1}^{n} b_{k}\left[a_{k}, a_{k+1}, \ldots, a_{n}, z\right],
\end{aligned}
$$


and

$$
g(z)=\prod_{k=1}^{n}\left|b_{k}\right|\left[\hat{a}_{k}, \hat{a}_{k+1}, \ldots, \hat{a}_{n}, z\right] .
$$

Hence, by (1.5), (1.6), (3.4) and (3.5), we obtain the inequality

$$
\begin{aligned}
|f(1-t+u t)| & =\prod_{k=1}^{n}\left|b_{k}\left[a_{k}, a_{k+1}, \ldots, a_{n},(1-t+u t)\right]\right| \\
& \leq \prod_{k=1}^{n}\left|b_{k}\right|\left[\hat{a}_{k}, \hat{a}_{k+1}, \ldots, \hat{a}_{n},(1-t+|u| t)\right] \\
& \leq g(1-t+w t)
\end{aligned}
$$

valid for $0 \leq t \leq 1$. Applying (3.7) to the right hand side of (3.6) we get

$$
\begin{aligned}
& \left|\left[a_{1}, a_{2}, \ldots, a_{m}\right]-\left[a_{1}, a_{2}, \ldots, a_{n}\right]\right| \leq|u-1| \int_{0}^{1} g(1+(w-1) t) d t \\
& =\frac{|u-1|}{w-1} \int_{0}^{1} g(1+(w-1) t) d(1+(w-1) t)=\frac{|u-1|}{w-1} \int_{1}^{w} g(z) d z \\
& =\frac{|u-1|}{w-1}\left(\left[\hat{a}_{1}, \hat{a}_{2}, \ldots, \hat{a}_{m}\right]-\left[\hat{a}_{1}, \hat{a}_{2}, \ldots, \hat{a}_{n}\right]\right)
\end{aligned}
$$

by (3.2) and (3.5). We observe that $w>1$ since $a_{n} \neq 1$ and hence $\hat{a}_{n}>1$. Moreover,

$$
\begin{aligned}
|u-1| & =\left|e^{b_{n+1}\left[a_{n+2}, \ldots, a_{m}\right]}-1\right|=\left|\sum_{k=1}^{\infty} \frac{1}{k !}\left(b_{n+1}\left[a_{n+2}, \ldots, a_{m}\right]\right)^{k}\right| \\
& \leq \sum_{k=1}^{\infty} \frac{1}{k !}\left(\left|b_{n+1}\right|\left[\hat{a}_{n+2}, \ldots, \hat{a}_{m}\right]\right)^{k}=w-1 .
\end{aligned}
$$

The statement of the theorem now follows from (3.8) and (3.9) by the Cauchy criterion for convergence.

Proof of Theorem 2. By Theorem 1 it suffices to consider real exponents $a_{n} \geq 1$. In this case the sequence $\left[a_{1}, a_{2}, a_{3}, \ldots\right]$ is monotonically increasing and it suffices to show that it is bounded. Define a sequence $\left\{c_{n}\right\}=\left\{c_{n}^{k_{0}+1}\right\}$ by

$$
c_{n}=\exp \left\{\frac{1+C_{n}^{k_{0}+1}}{e}\right\} \quad\left[n \geq n_{k_{0}+1}\right],
$$


where $C^{k_{0}+1}$ and $n_{k_{0}+1}$ are defined in the statement of Lemma 2 . Setting $C_{n}=C^{k_{0}+1}$, we have, by (2.5), (2.1) and (1.7),

$$
\begin{aligned}
1+\log \log c_{n} & =\log \left(1+C_{n}\right)=C_{n}+O\left(C_{n}^{2}\right)>\frac{1}{2} \sum_{j=0}^{k_{0}} l_{j}^{2}(n) \\
& \geq 1+\log \log a_{n},
\end{aligned}
$$

for $n \geq n_{0}$ sufficiently large in terms of $k_{0}$ as we may assume. Therefore for $n \geq n_{0}, a_{n} \leq c_{n}$ and hence

$$
\left[a_{n_{0}}, a_{n_{0}+1}, \ldots, a_{n}\right] \leq\left[c_{n_{0}}, c_{n_{0}+1}, \ldots, c_{n}\right] .
$$

Thus it suffices to show that the infinite exponential $\left[c_{n_{0}}, c_{n_{0}+1}, c_{n_{0}+2}, \ldots\right]$ converges. By Lemma 1 this in term is equivalent to the existence of a sequence $S_{n}, n=n_{0}, n_{0}+1, n_{0}+2, \ldots$, satisfying $S_{n} \geq-1$ and

$$
1+S_{n} \geq\left(1+C_{n}\right) e^{S_{n+1}} .
$$

But by Lemma 2

$$
1+C_{n}=1+C_{n}^{k_{0}+1}<1+X_{n}^{k_{0}+1}=\left(1+T_{n}^{k_{0}+1}\right) e^{-T_{n+1}^{k_{0}+1}} .
$$

Hence (3.10) is satisfied with $S_{n}=T_{n}^{k_{0}+1}, n \geq n_{0}$. This completes the proof of the theorem.

Proof of Theorem 3 . We argue by contradiction. Suppose to the contrary that the infinite exponential $\left[a_{1}, a_{2}, a_{3}, \ldots\right]$ is convergent. Then, since $a_{n}>1$, so is $\left[a_{n}, a_{n+1}, a_{n+2}, \ldots\right]$ for any $n \geq 1$. Let us denote the limit of such an infinite exponential by $e^{1+S_{n}}$. Let us also define $A_{n}$ by

$$
a_{n}=\exp \left\{\frac{1+A_{n}}{e}\right\}
$$

Then

$$
e^{1+S_{n}}=\left[a_{n}, e^{1+S_{n+1}}\right]=e^{\left(1+A_{n}\right) e^{S_{n+1}}} .
$$

In the remainder of this proof we will use $n$ to denote an integer satisfying $n \geq n_{0}$. For such $n$, it is immediate from (1.8) that 
$A_{n}>0$, since $a_{n}>e^{1 / e}$. Moreover, by (3.11), (1.8), (2.1), (2.5) and (2.7), we have

(3.13) $A_{n} \geq \log \left(1+A_{n}\right)=1+\log \log a_{n} \geq C_{n}^{k_{0}}+\frac{\epsilon}{2} l_{k_{0}}^{2}(n)$

$$
>C_{n}^{k_{0}+1}>X_{n}^{k_{0}}
$$

for $n \geq n_{0}$ sufficiently large in terms of $k_{0}$ and $\epsilon$, as we may assume. This gives

$$
a_{n}>x_{n}^{k_{0}}
$$

where $x_{n}^{k_{0}}$ is defined by (2.14). Therefore, by the definition of $S_{n}$ and Lemma 3, we get

$$
S_{n}>T_{n}^{k_{0}}
$$

We set

$$
R_{n}=S_{n}-T_{n}^{k_{0}}
$$

and

$$
B_{n}=A_{n}-X_{n}^{k_{0}}
$$

Then by (3.14)

$$
R_{n}>0
$$

and by (3.13), (2.13), (2.5) and (2.1)

$(3.16)$

$$
\begin{aligned}
B_{n} & \geq C_{n}^{k_{0}}+\frac{\epsilon}{2} l_{k_{0}}^{2}(n)-X_{n}^{k_{0}}=\frac{\epsilon}{2} l_{k_{0}}^{2}(n)+O_{k_{0}}\left(\frac{1}{n^{3}}\right) \\
& =\epsilon\left(C_{n}^{k_{0}}-C_{n}^{k_{0}-1}\right)+O_{k_{0}}\left(\frac{1}{n^{3}}\right) \\
& =\epsilon\left(X_{n}^{k_{0}}-X_{n}^{k_{0}-1}\right)+O_{k_{0}}\left(\frac{1}{n^{3}}\right) \\
& >\frac{\epsilon}{2}\left(X_{n}^{k_{0}}-X_{n}^{k_{0}-1}\right),
\end{aligned}
$$

for $n \geq n_{0}$ sufficiently large in terms of $k_{0}$ and $\epsilon$. Now, by (3.12), (2.6), (3.15) and (3.16), we have

$$
\begin{aligned}
1 & +T_{n}^{k_{0}}+R_{n} \\
& =\left(1+X_{n}^{k_{0}}+B_{n}\right) e^{T_{n+1}^{k_{0}}+R_{n+1}}=\left(1+T_{n}^{k_{0}}\right) e^{R_{n+1}}+B_{n} e^{T_{n+1}^{k_{0}}+R_{n+1}} \\
& >\left(1+T_{n}^{k_{0}}\right)\left(1+R_{n+1}\right)+\frac{\epsilon}{2}\left(\left(1+X_{n}^{k_{0}}\right)-\left(1+X_{n}^{k_{0}-1}\right)\right) e^{T_{n+1}^{k_{0}}} \\
& =\left(1+T_{n}^{k_{0}}\right)\left(1+R_{n+1}\right)+\frac{\epsilon}{2}\left(T_{n}^{k_{0}}-T_{n}^{k_{0}-1}\right) .
\end{aligned}
$$


This together with (3.15) and (2.4) yield

$$
R_{n}>R_{n+1}+\frac{\epsilon}{2} l_{k_{0}}(n-1) .
$$

Hence we obtain the bound

$$
R_{n}>\frac{\epsilon}{2} \sum_{m=n-1}^{\infty} l_{k_{0}}(m)
$$

But the last assertion is absurd in view of the definition (2.1) of $l_{k_{0}}(m)$. This contradicts our assumption and thus completes the proof of the theorem.

\section{REFERENCES}

[B] D.F. Barrow, Infinite exponentials, American Math. Monthly, 43 (1936), 150-160.

[E] Leonhard Euler, De formulis exponentialibus replicatis, Acta Academiae Scientiarum Petropolitanae, 1 (1778), 38-60.

[G] Richard Guy, Western Number Theory Problems, 1991-12-19\&22, 1992.

[K] Arthur Knoebel, Exponentials reiterated, American Math. Monthly, 88 (1981), 235-252.

[R] S. Ramanujan, Notebooks, Vol. 2, Tata Institute of Fundamental Research, Bombay, 1957.

[S] Donald L. Shell, On the convergence of infinite exponentials, Proceedings of the American Math. Society, 13 (1962), 678-681.

[T] W.J. Thron, Convergence of infinite exponentials with complex elements, Proceedings of the American Math. Society, 8 (1957), 1040-1043.

Received October 26, 1992 and in revised form March 4, 1994. I would like to thank Professor Adolf Hildebrand for both suggesting this problem and for his help which led to the present version of this paper.

UNIVERSITY OF NEVADA

LAS VEGAS, NV 89154-4020

E-mail address: bachman@nevada.edu 



\title{
PACIFIC JOURNAL OF MATHEMATICS
}

Founded by E. F. Beckenbach (1906-1982) and F. Wolf (1904-1989)

\section{EDITORS}

Sun-Yung Alice Chang (Managing Editor)

University of California

Los Angeles, CA 90095-1555

pacific@math.ucla.edu

F. Michael Christ

University of California

Los Angeles, CA 90095-1555

christ@math.ucla.edu

\section{Thomas Enright}

University of California

San Diego, La Jolla, CA 92093

tenright@ucsd.edu

Nicholas Ercolani

University of Arizona

Tucson, AZ 85721

ercolani@math.arizona.edu
Robert Finn

Stanford University

Stanford, CA 94305

finn@gauss.stanford.edu

Vaughan F. R. Jones

University of California

Berkeley, CA 94720

vfr@math.berkeley.edu

Steven Kerckhoff

Stanford University

Stanford, CA 94305

spk@gauss.stanford.edu
Martin Scharlemann

University of California

Santa Barbara, CA 93106 mgscharl@math.ucsb.edu

Gang Tian

Courant Institute

New York University

New York, NY 10012-1100

tiang@taotao.cims.nyu.edu

V. S. Varadarajan

University of California

Los Angeles, CA 90095-1555

vsv@math.ucla.edu

\section{SUPPORTING INSTITUTIONS}

\section{CALIFORNIA INSTITUTE OF TECHNOLOGY NEW MEXICO STATE UNIVERSITY \\ OREGON STATE UNIVERSITY \\ STANFORD UNIVERSITY \\ UNIVERSITY OF ARIZONA \\ UNIVERSITY OF BRITISH COLUMBIA \\ UNIVERSITY OF CALIFORNIA \\ UNIVERSITY OF HAWAII}

\author{
UNIVERSITY OF MONTANA \\ UNIVERSITY OF NEVADA, RENO \\ UNIVERSITY OF OREGON \\ UNIVERSITY OF SOUTHERN CALIFORNIA \\ UNIVERSITY OF UTAH \\ UNIVERSITY OF WASHINGTON \\ WASHINGTON STATE UNIVERSITY
}

The supporting Institutions listed above contribute to the cost of publication of this Journal, but they are not owners or publishers and have no responsibility for its contents or policies.

Manuscripts must be prepared in accordance with the instructions provided on the inside back cover.

The Pacific Journal of Mathematics (ISSN 0030-8730) is published monthly except for July and August. Regular subscription rate: $\$ 215.00$ a year (10 issues). Special rate: $\$ 108.00$ a year to individual members of supporting institutions.

Subscriptions, orders for back issues published within the last three years, and changes of subscribers address should be sent to Pacific Journal of Mathematics, P.O. Box 4163, Berkeley, CA 94704-0163, U.S.A. Prior back issues are obtainable from Kraus Periodicals Co., Route 100, Millwood, NY 10546.

The Pacific Journal of Mathematics at the University of California, c/o Department of Mathematics, 981 Evans Hall Berkeley, CA 94720 (ISSN 0030-8730) is published monthly except for July and August. Second-class postage paid at Berkeley, CA 94704, and additional mailing offices. POSTMASTER: send address changes to Pacific Journal of Mathematics, P.O. Box 6143, Berkeley, CA 94704-0163.

\author{
PUBLISHED BY PACIFIC JOURNAL OF MATHEMATICS at University of California, \\ Berkeley, CA 94720, A NON-PROFIT CORPORATION \\ This publication was typeset using AMS-LATEX, \\ the American Mathematical Society's TEX macro system. \\ Copyright (C) 1995 by Pacific Journal of Mathematics
}




\section{PACIFIC JOURNAL OF MATHEMATICS}

Volume $169 \quad$ No. $2 \quad$ June 1995

On Banach spaces $Y$ for which $B(C(\Omega), Y)=K(C(\Omega), Y)$

201

SHAMIM ISMAIL ANSARI

Convergence of infinite exponentials

219

GENNADY BACHMAN

Cohomologie d'intersection modérée. Un théorème de de Rham

235

Bohumil Cenkl, Gilbert Hector and Martintxo

SARALEGI-ARANGUREN

Kleinian groups with an invariant Jordan curve: $J$-groups

291

Ruben A. Hidalgo

Multiplicative functions on free groups and irreducible representations 311

M. GABRiella KuHN and Tim STEGer

A Diophantine equation concerning finite groups

MAOHUA LE

Nilpotent characters

343

GABRIEL NAVARRO

Smooth extensions and quantized Fréchet algebras

XiaOlU WANG 